\title{
Saliva Versus Plasma Bioequivalence of Rusovastatin in Humans: Validation of Class III Drugs of the Salivary Excretion Classification System
}

\author{
Nasir Idkaidek • Tawfiq Arafat
}

Published online: 11 February 2015

(C) The Author(s) 2015. This article is published with open access at Springerlink.com

\begin{abstract}
Bioequivalence of rusovastatin in healthy human volunteers was done using saliva and plasma matrices in order to investigate the robustness of using saliva instead of plasma as a surrogate for bioequivalence of class III drugs according to the salivary excretion classification system (SECS). Saliva and plasma samples were collected for $72 \mathrm{~h}$ after oral administration of rusovastatin $40 \mathrm{mg}$ to 12 healthy humans. Saliva and plasma pharmacokinetic parameters were calculated by non-compartmental analysis. Analysis of variance, $90 \%$ confidence intervals, and intra-subject and inter-subject variability values of pharmacokinetic parameters were calculated using Kinetica program V5. Human effective intestinal permeability was also calculated by SimCYP program V13. Rusovastatin falls into class III (high permeability/ low fraction unbound to plasma proteins) and hence was subjected to salivary excretion. A correlation coefficient of 0.99 between saliva and plasma concentrations, and a saliva/plasma concentration ratio of 0.175 were observed. The $90 \%$ confidence limits of area under the curve $\left(\mathrm{AUC}_{\text {last }}\right)$ and maximum concentration $\left(C_{\max }\right)$ showed similar trends in both saliva and plasma. On the other hand, inter- and intra-subject variability values in saliva were higher than in plasma, leading to the need for a slightly higher number of subjects to be used in saliva studies. Non-invasive saliva sampling instead of the invasive plasma sampling method can be used as a surrogate for bioequivalence of SECS class III drugs when an adequate sample size is used.
\end{abstract}

N. Idkaidek $(\bowtie) \cdot T$. Arafat

College of Pharmacy, University of Petra, PO Box 961343,

Amman, Jordan

e-mail: nidkaidek@uop.edu.jo

\section{Introduction}

Salivary excretion of some drugs has been reported previously as a good indicator for drug bioavailability, therapeutic drug monitoring, pharmacokinetics and also drug abuse. Saliva sampling is a simple, non-invasive and cheap method compared with plasma sampling, with no contamination risk [1,2]. The rules of drug protein binding and membrane permeability on salivary excretion were previously investigated for several drugs, where a Salivary Excretion Classification System (SECS) was proposed as shown in Table 1 [3]. High intestinal permeability corresponds to fraction absorption $\left(F_{\mathrm{a}}\right)>0.9$, while high protein binding corresponds to low fraction unbound (fu) of $<0.1$ $[4,5]$. According to SECS, Class I drugs of high intestinal permeability and low protein binding, such as paracetamol, are subject to salivary excretion. Class II drugs of low permeability and low protein binding, such as metformin, are subject to salivary excretion since low permeability is counterbalanced by low protein binding. Class III drugs of high intestinal permeability and high protein binding, such as rusovastatin, are subject to salivary excretion since high protein binding is counterbalanced by high permeability. Class IV drugs of low intestinal permeability and high protein binding, such as montelukast, are not subject to salivary excretion [3].

\section{Objectives}

The objective of this study is to investigate the robustness of using a non-invasive saliva sampling method instead of a plasma sampling method as a surrogate for bioequivalence of SECS class III drugs that are excreted in saliva, using rusovastatin as a model drug. 
Table 1 Salivary excretion classification system (SECS) according to drug permeability $\left(P_{\text {eff }}\right)$ and fraction unbound to plasma proteins (fu)

\begin{tabular}{llll}
\hline Class & \multicolumn{2}{l}{ Parameter } & \\
\cline { 2 - 4 } & $P_{\text {eff }}$ & fu & Salivary excretion \\
\hline Class I & High & High & Yes \\
Class II & Low & High & Yes \\
Class III & High & Low & Yes \\
Class IV & Low & Low & No \\
\hline
\end{tabular}

\section{Methods}

\subsection{Study Design}

Saliva pharmacokinetics under a fasted state, in 12 healthy human volunteers after signing informed consent, were compared with plasma pharmacokinetics in a two-way, cross-over design study with washout time between phases of 1 week. Medical history, vital signs, physical examination and laboratory safety test results showed no evidence of clinically significant deviation from normal medical condition as evaluated by the clinical investigator. The pilot bioequivalence study was conducted as per the International Conference on Harmonisation of Technical Requirements for Registration of Pharmaceuticals for Human Use (ICH), Good Clinical Practice (GCP), and Helsinki declaration guidelines after Institutional Review Board (IRB) of Jordan Center of Pharmaceutical Research and Jordan FDA approvals.

An oral dose of $40 \mathrm{mg}$ test product rusovastatin (Batch no. RD-04F13) or reference product (Batch no. KG115) with $240 \mathrm{ml}$ of water was given after $10 \mathrm{~h}$ overnight fasting without dietary restrictions. Then resting saliva (without stimulation) and plasma samples were collected at $0,1,2,2.5,3,3.5,4,4.5,5,5.5,6,7$, $8,12,24,48$ and truncated at $72 \mathrm{~h}$ during each study phase. Thorough rinsing of the mouth was done after dosing to avoid contamination of saliva samples with any drug residues.

\subsection{Assay Methodology}

All saliva and plasma samples were deep frozen until assayed by a validated liquid chromatography-mass spectrometry (LC-MS) assay method. Intra-day coefficient of variation $(\mathrm{CV})$ was $14.26 \%$, inter-day accuracy range was $91.76-102.80 \%$, inter-day precision range was $2.21-13.41 \%$ and linear range was $0.5-40.00 \mathrm{ng} / \mathrm{mL}$.

\subsection{Data Analysis}

\subsubsection{Pharmacokinetic Parameters}

Individual pharmacokinetic parameters for drug concentration in both saliva and plasma samples were calculated by non-compartmental analysis (NCA) using Kinetica program V5. Pharmacokinetic parameters were area under the concentration curves to last collection time $\left(\mathrm{AUC}_{t}\right)$, maximum measured concentration $\left(C_{\max }\right)$ and time to maximum concentration $\left(T_{\max }\right)$. Elimination parameters of half life, elimination rate constant and $\mathrm{AUC}_{\infty}$ were not calculated since sampling was truncated at $72 \mathrm{~h}$. Descriptive statistics and pharmacokinetic parameters were undertaken using Microsoft Excel.

\subsubsection{Dimensional and Correlation Analysis}

Saliva versus plasma concentrations up to median $T_{\max }$ values were correlated by linear regression using Microsoft Excel. Conversely, dimensional analysis was done on an individual basis, and then averages were statistically compared using a $t$ test. Dimensional analysis offers the advantage of more clear comparisons since ratios are unitless. The following dimensionless ratios were calculated:

$$
\begin{aligned}
\mathrm{AUC}^{*} & =\text { saliva } \mathrm{AUC}_{t} / \text { plasma } \mathrm{AUC}_{t} \\
T_{\max }{ }^{*} & =\text { saliva } T_{\max } / \text { plasma } T_{\max } \\
C_{\max }{ }^{*} & =\text { saliva } C_{\max } / \text { plasma } C_{\max } \\
C^{*} & =\text { saliva concentration } / \text { plasma concentration }=C_{\mathrm{s}} / C_{\mathrm{p}} .
\end{aligned}
$$

\subsubsection{Bioequivalence Analysis}

Analysis of variance (ANOVA), $90 \%$ confidence intervals, and inter-subject and intra-subject variability estimates for primary pharmacokinetic parameters $\left(\mathrm{AUC}_{t}\right.$ and $C_{\max }$ ) for test/reference ratios after logarithmic transformation were calculated by Kinetica program V5. The ANOVA model included the following sources of variations: phase, treatment, sequence and subject nested in sequence.

\subsubsection{Optimized Effective Intestinal Permeability}

Effective intestinal permeability $\left(P_{\text {eff }}\right)$ values were estimated by the Nelder-Mead algorithm of the parameter estimation module using the SimCYP program [6]. The Nelder-Mead method, which is also called the downhill simplex method, is a commonly used nonlinear optimization algorithm. This involved searching for the best parameter values that produce plasma concentration that 
matches the actual plasma concentration at the same time. The objective function is the weighted sum of squared differences of observed and model predicted values. Polar surface area (PSA) was used first, using SimCYP, to predict an initial estimate of $P_{\text {eff. }}$.

\section{Results and Discussion}

Rusovastatin falls into SECS class III with high permeability $\left(F_{\mathrm{a}}=1\right)$ and high protein binding $(\mathrm{fu}=0.1)$ [3]. It showed good salivary excretion with a correlation coefficient of 0.99 between saliva and plasma concentrations up to median $T_{\max }$ values of plasma profiles as shown in Figs. 1 and 2. Assuming a one-compartment linear model, the salivary excretion rate is dependent on plasma drug concentration. This explains the close behavior of the sal-

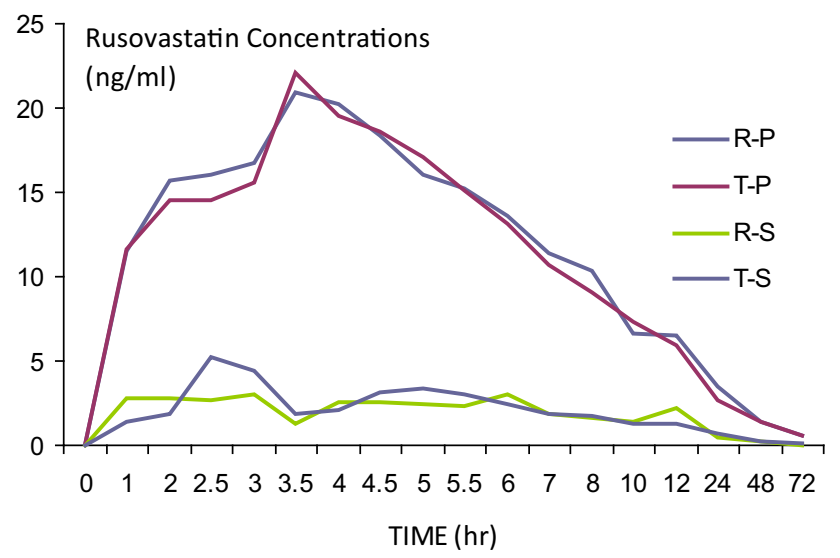

Fig. 1 Plasma and saliva of rusovastatin mean concentrations (ng/ $\mathrm{mL}$ ) after $40 \mathrm{mg}$ oral dose iva and plasma profiles. This result is in agreement with a previous study with rusovastatin [3]. Pharmacokinetic parameters in saliva and plasma for rusovastatin are summarized in Table 2. $\mathrm{AUC}_{t}$ and $C_{\max }$ parameter values were lower in saliva as compared with plasma. However, $T_{\max }$ was longer in saliva, suggesting a lag time between plasma and saliva compartments due to drug distribution/redistribution processes in the body.

In addition, dimensional analysis results shown in Table 3 indicated lower bioavailability in saliva, yet no significant difference was observed between test and reference ratios. This can be related to the effect of high rusovastatin membrane effective permeability and high protein binding, leading to low saliva/plasma ratios. Moreover, $\mathrm{AUC}^{*}$ and $C_{\max }{ }^{*}$ values were in close agreement with $C^{*}$ values.

On the other hand, bioequivalence metrics and variability percentages for primary pharmacokinetic parameters were calculated in both saliva and plasma as shown in Tables 4 and 5 . The $90 \%$ confidence intervals did not fall within the $80-125 \%$ acceptance range as per the guideline (7), which is consistent with the high variability values observed in this pilot study. Hence, and due to higher intersubject and intra-subject variability value in saliva as compared with plasma, more subjects are needed in the pivotal study using saliva matrix compared with plasma matrix. The optimum sample size was recalculated using Study Result program V1. Forty-six and 48 human subjects will be needed to account for 46.4 and $48 \%$ intra-subject variability of $C_{\max }$ in plasma and saliva, respectively. ANOVA $p$ values shown in Table 6 indicated insignificant differences $(p>0.05)$ in all sources except subject (sequence) in saliva, which could be due to the small sample size used in this pilot study.
Fig. 2 Correlation of saliva and plasma rusovastatin mean concentrations

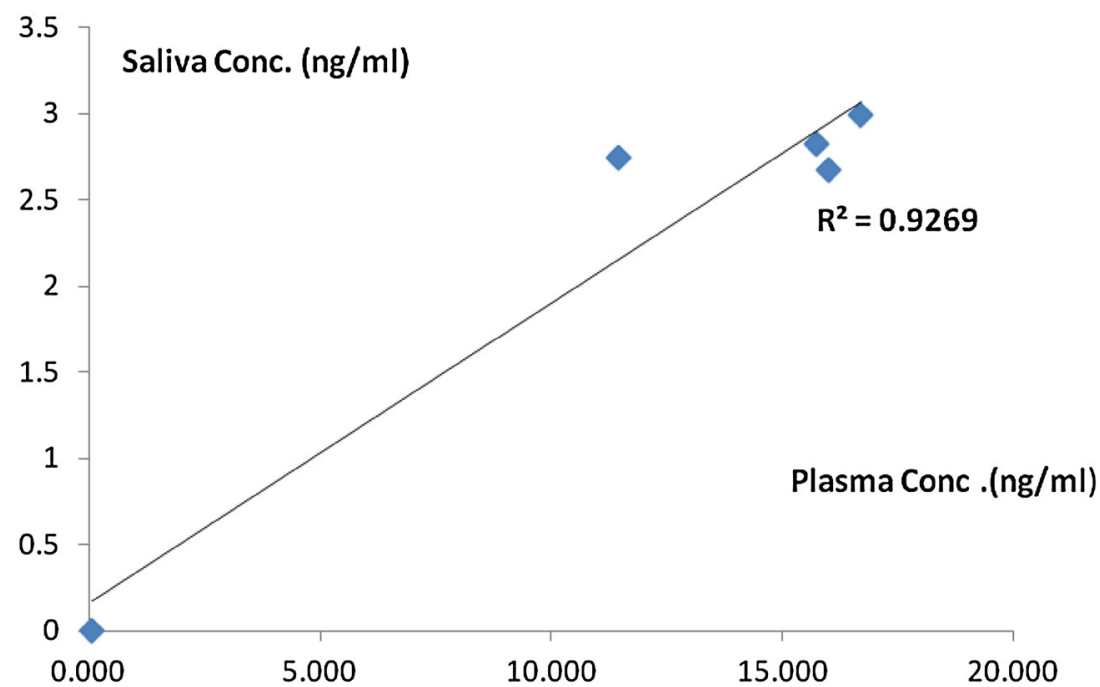


Table 2 Saliva and plasma mean (\% CV) pharmacokinetic parameters after a 40-mg oral dose of rusovastatin tablet to 12 healthy volunteers

\begin{tabular}{lccrr}
\hline Parameter & \multicolumn{1}{l}{ Matrix } & & & \\
\cline { 2 - 5 } & Saliva test & Saliva reference & Plasma test & Plasma reference \\
\hline $\mathrm{AUC}_{t}(\mathrm{ng} / \mathrm{mL} \mathrm{h})$ & $41.54(98)$ & $48.78(89)$ & $264.05(41)$ & $281.92(33)$ \\
$\mathrm{C}_{\max }(\mathrm{ng} / \mathrm{mL})$ & $6.42(141)$ & $6.78(90)$ & $24.18(42)$ & $22.74(49)$ \\
$\mathrm{T}_{\max }(\mathrm{h})$ & $4.5(39)$ & $4.25(66)$ & $3.79(13)$ & $3.54(66)$ \\
\hline
\end{tabular}

$A U C_{t}$ area under the concentration curves to last collection time, $C_{\max }$ maximum measured concentration, $C V$ coefficient of variation, $T_{\max }$ time to maximum concentration

Table 3 Dimensional analysis values of pharmacokinetic parameters

\begin{tabular}{llll}
\hline Parameter & Test & Reference & $p$ value \\
\hline AUC* $^{*}$ & 0.17 & 0.17 & 0.92 \\
$C_{\max }{ }^{*}$ & 0.25 & 0.35 & 0.56 \\
$T_{\max }{ }^{*}$ & 1.23 & 1.47 & 0.15 \\
$C^{*}$ & 0.17 & 0.18 & 0.46 \\
\hline
\end{tabular}

$\mathrm{AUC}^{*}=$ saliva $\quad \mathrm{AUC}_{t} /$ plasma $\quad \mathrm{AUC}_{t} ; T_{\max }{ }^{*}=$ saliva $T_{\max } /$ plasma $T_{\max } ; C_{\max }{ }^{*}=$ saliva $C_{\max } /$ plasma $C_{\max } ; C^{*}=$ saliva concentration/ plasma concentration $=C_{\mathrm{s}} / C_{\mathrm{p}}$

$A U C_{t}$ area under the concentration curves to last collection time, $C_{\max }$ maximum measured concentration, $T_{\max }$ time to maximum concentration

Table 4 Bioequivalence metrics of primary pharmacokinetic parameters after log transformation

\begin{tabular}{llr}
\hline Parameter & \multicolumn{1}{c}{ Saliva $^{*}$} & \multicolumn{1}{c}{ Plasma $^{*}$} \\
\hline AUC $_{t}$ & $81.8(57.7-115.9)$ & $90(72.7-111.4)$ \\
$C_{\max }$ & $87.4(61.3-124.8)$ & $107.5(76.3-151.6)$ \\
\hline
\end{tabular}

$\mathrm{AUC}_{t}$ area under the concentration curves to last collection time, $C_{\max }$ maximum measured concentration

* Point estimate and $90 \%$ confidence intervals (lower limit-upper limit)

Table 5 Saliva, plasma inter-subject and intra-subject variability values of primary pharmacokinetic parameters after log transformation

\begin{tabular}{lll}
\hline Parameter & Inter-subject CV \% & Intra-subject CV \% \\
\hline AUC $_{t}$ & $13.7,5.2$ & $47.2,28.8$ \\
$C_{\max }$ & $32.8,15.2$ & $48.0,46.4$ \\
\hline
\end{tabular}

$A U C_{t}$ area under the concentration curves to last collection time, $C_{\max }$ maximum measured concentration

Mean concentration profiles of the reference product were used to estimate the effective intestinal permeability values in plasma and saliva. Figure 3 shows observed
Table 6 ANOVA $p$ values $\left(\mathrm{AUC}_{t}, C_{\max }\right)$ in plasma and saliva

\begin{tabular}{lll}
\hline Source & Plasma & Saliva \\
\hline Phase & $0.245,0.482$ & $0.885,0.302$ \\
Treatment & $0.391,0.709$ & $0.320,0.509$ \\
Sequence & $0.355,0.469$ & $0.178,0.812$ \\
Subject (sequence) & $0.075,0.486$ & $0.002^{*}, 0.002^{*}$ \\
\hline
\end{tabular}

ANOVA analysis of variance, $A U C_{t}$ area under the concentration curves to last collection time, $C_{\max }$ maximum measured concentration * Significant difference observed since $p<0.05$

versus SimCYP-predicted concentration profiles with correlation coefficients of $0.85-0.87$, indicating good fitting of observed concentrations. Optimized effective permeability coefficients were 68.17 and $68.30 \times 10^{-4}$ $\mathrm{cm} / \mathrm{sec}$ in plasma and saliva, respectively, which is in agreement with the previous value of $74.33 \times 10^{-4} \mathrm{~cm} /$ sec [3]. This confirms our previous finding that effective permeability and protein binding are major key factors in salivary excretion and our previous assumption that intestinal permeability is similar to salivary mucosal permeability [3].

From a regulatory point of view, the US FDA guidance for industry stated "The statutory definitions of BA and $\mathrm{BE}$, expressed in terms of rate and extent of absorption of the active ingredient or moiety to the site of action, emphasize the use of pharmacokinetic measures in an accessible biological matrix such as blood, plasma, and/or serum to indicate release of the drug substance from the drug product into the systemic circulation" [7]. Hence, from the data collected for drugs in SECS class III such as rusovastatin, there is a potential in bioavailability/bioequivalence (BA/BE) studies for saliva to be considered as a surrogate for plasma concentration. This line of research can help validate the newly proposed salivary excretion classification system. The use of saliva instead of plasma in such studies makes them non-invasive, easy and with a lower clinical burden. 
Fig. 3 Observed versus SimCYP-predicted rusovastatin concentrations of the reference product

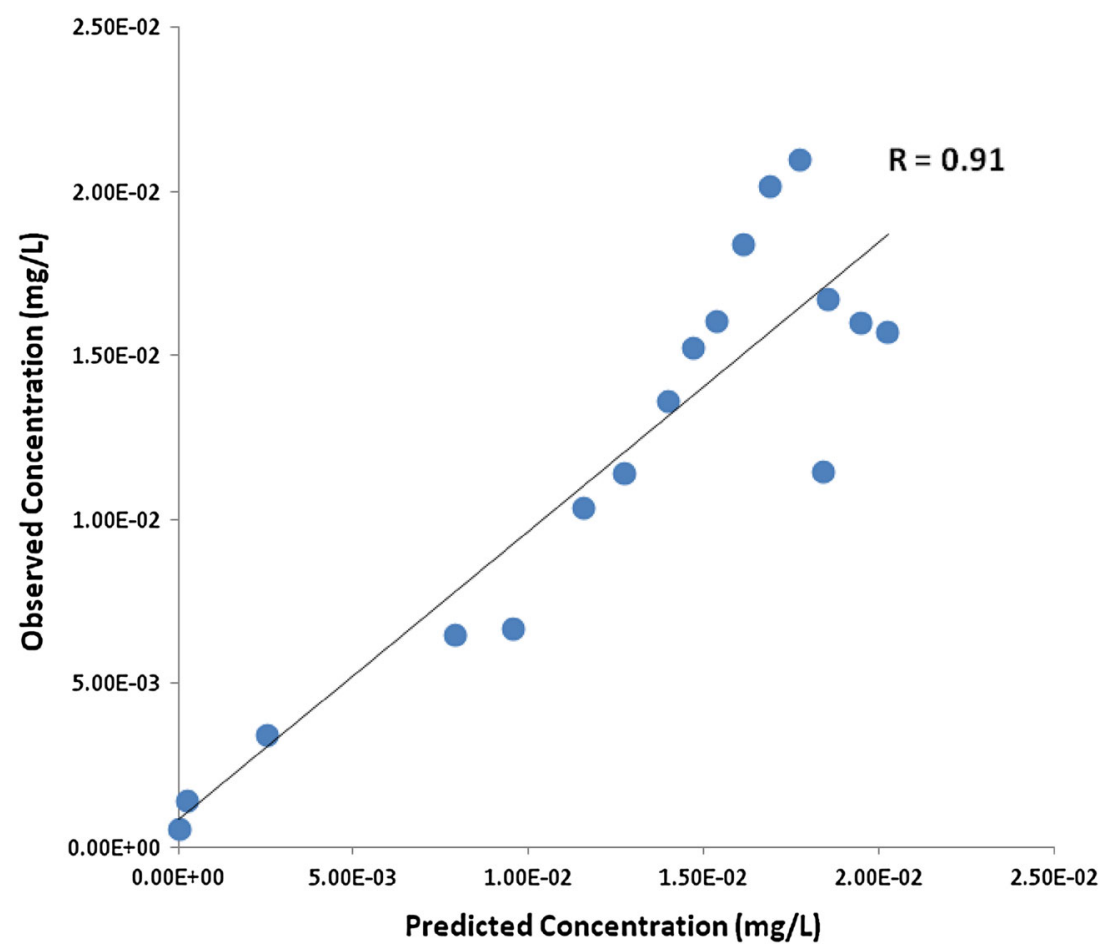

\section{Future Work}

More research studies of candidate drugs that fall into classes I, II and III are needed in order to compare saliva versus plasma bioavailability and bioequivalence; and to demonstrate SECS robustness.

Acknowledgments This research was funded by the University of Petra. Kinetica was used under academic license from Innaphase Ltd, France (Lic. \# K 201009). SimCYP program was used under academic license from SimCYP Ltd, Sheffield, UK (Lic. \# CLCLIDAKDI-LEEE-FECI).

Conflict of interest Authors of the manuscript do not have any conflicts of interest to declare.

Open Access This article is distributed under the terms of the Creative Commons Attribution Noncommercial License which permits any noncommercial use, distribution, and reproduction in any medium, provided the original author(s) and the source are credited.
2. Ruiz ME, Conforti P, Fagiolino P, Volonté MG. The use of saliva as a biological fluid in relative bioavailability studies: comparison and correlation with plasma results. Biopharm Drug Dispos. 2010;31(8-9):476-85.

3. Idkaidek Nasir, Arafat Tawfiq. Saliva versus plasma pharmacokinetics: theory and application of a salivary excretion classification system. Mol Pharmaceutics. 2012;9:2358-63.

4. Amidon GL, Lennernäs H, Shah VP, Crison JR. A theoretical basis for a biopharmaceutics drug classification: the correlation of in vitro drug product dissolution and in vivo bioavailability. Pharm Res. 1995;12(3):413-20.

5. Sunil S Jambhekar and Philip J Breen. Basic Pharmacokinetics, First Edition. London: Pharmaceutical Press, 2009.

6. Jamei M, Turner D, Yang J, Neuhoff S, Polak S, RostamiHodjegan A, Tucker G. Population-based mechanistic prediction of oral drug absorption. AAPS J. 2009;11(2):225-37.

7. Guidance for Industry: bioavailability and bioequivalence studies for orally administered drug products-general considerations. March 2003. Division of Drug Information, Center for Drug Evaluation and Research, Food and Drug Administration 5600 Fishers Lane Rockville, MD 20857, USA.

\section{References}

1. Gorodischer R, Koren G. Salivary excretion of drugs in children: theoretical and practical issues in therapeutic drug monitoring. Dev Pharmacol Ther. 1992;19(4):161-77. 